\title{
Implementation of the Anti-Quackery Mandate Punjab Healthcare Commission: Challenges and Limitations
}

\author{
AIN UL MOMINA ${ }^{1}$, RUBEENA ZAKAR ${ }^{2}$ \\ ${ }^{1}$ Associate Professor, King Edward Medical University \\ ${ }^{2}$ Professor/Director, Department of Public Health, University of the Punjab \\ Correspondence to Dr. Ain ul Momina, email: ainmomina@gmail.com
}

\begin{abstract}
Background: Medical quackery is a big challenge forhealthcare system in Pakistan. This menace was particularly widespread in Punjab, where a regulatory body called the Punjab Healthcare Commission (PHC) was formulatedin 2010. PHC established an Anti-Quackery Cell with the objective of curbing the spread of quackery in the Province. Aim: To find out the challenges that the Anti-Quackery Cell encountered to control quackery.

Method: The current study applied a qualitative research method, with a focus on exploratory research design. A total of $20 \mathrm{PHC}$ and Anti-Quackery Cell officials were identified by adopting a purposive sampling technique. An interview guide was used to collect data from respondents. An inductive as well as deductive approach was used to extract themes from the data and generate findings.

Results: The findings of the study show that the lack of political and administrative will was the most significant challenge to control quackery in Pakistan. Furthermore, inappropriate staff selection, untrained staff, lack of proper legislation and the deep roots quackery in society were other factors that weaken the capacity of the AntiQuackery Cell.

Conclusion: The study concludes that it is very difficult to successfully curb quackery due to the relevance of the practice with the socio-economic and cultural context of Pakistan. However, proper legislation, professional development of staff and strong political and administrative commitment are the way forward.

Keywords: PHC, Quackery, healthcare system
\end{abstract}

\section{INTRODUCTION}

A healthcare system is one of the most important threads in the social fabric of society. Such an institution has an expansive scope and a mandate to promote the well-being of individuals. Across the world, healthcare systems are formulated on the basis of modern techniques and professionalism ${ }^{1}$. However, in developing countries,these systems encounter multiple issues that hamper health service delivery, such as quackery.

The prevalence of quackery is very high among developingcountries as compared to developed countries ${ }^{2}$. Taking the case of Pakistan, quackery is pervasive and deeply ingrained in society. It is practiced in many forms in Pakistan, from 'quacks' who practice allopathic medicine without the required qualification to informal healthcare providers who roam freely in academic circles and the public at large,depending on the form of services provided ${ }^{3}$. Quacks are found in rural and urban areas of the country alike.

The prevalence of quackery is particularly omnipresent in Punjab. In 2008, the Punjab Health Sector Reform Program estimated that 7079 quacks were practicing in the Province ${ }^{4}$. The harm done by quackery is multifarious, ranging from"economic harm (to individuals and society), direct harm (injury, permanent injury, death), indirect harm (injury, permanent injury, death), psychological harm, harm to society, and harm to professionalism"5. All these bodily and psychosocial harms are detrimental to the health of individuals and society, which is why it is imperative to control the spread of quackery in Pakistan.

Received on 09-04-2021

Accepted on 19-08-2021
Keeping in view the severity of the issue, the Government of Punjab established a regulatory body calledthe Punjab Health Care Commission (PHC) in 2010. The main goal of PHC is to "improve quality of healthcare services and ban quackery in Punjab in all its forms and manifestations". According to Section 2 of the PHC Act, 2010, a 'quack' means a pretender providing healthcare services without registration with the Pakistan Medical and Dental Council, Council for Tibb, Council for Homeopathy or Nursing Council ${ }^{6}$. Furthermore, under Section 4 of the same Act, one of the regulatory functions of $\mathrm{PHC}$ is "taking necessary steps to ban quackery". The anti-quackery mandate of $\mathrm{PHC}$ is as follows:

1. Registration of all healthcare service providers as an exclusion strategy

2. Support legislative reforms

3. Institutionalization of administrative set up at PHC establish Anti-Quackery Cell

4. Advocacy through behavior change communication

5. Inter-sector coordination for enforcement of antiquackery activities

6. Alternative solutions for unmet needs of patients

7. Consultations with key stakeholders

However, across the globe, the success of regulatory bodies in controlling quackery is not up to the mark. Developing countries like Kenya,as well as several developed countries, are expressing doubts on the effectiveness of regulation to limit quackery and enhance quality of healthcare ${ }^{7}$.

The current study has been conducted "to explore challenges faced byPHC in realizing its Anti-Quackery Mandate". Despite PHC's efforts to curtail quackery, the issue remains rampant in the province. The findings of this study are beneficial for policymakers to evaluate their 
approach and find new ways to deal with the issue, as well as for field workers to address ongoingissues that hinder the effective elimination of quackery in Punjab.

\section{METHODOLOGY}

This study adopted a qualitative research design to inquire challenges and barriers to implement the anti-quackery mandate of PHC. An inductive as well as deductive approach was used to extract themes and reach a valid conclusion ${ }^{8}$.

After permission from departmental IRB, a purposive sampling technique was adopted as it is an important way of identifying respondents for an in-depth qualitative understanding of any social phenomenon. The use of this technique has been validated in qualitative research owing to following three major reasons which apply to the current study ${ }^{9}$ :

It is an important way of reaching a specialized population. It helps in the identification of respondents with valid and enriched information. It is best suited for in-depth understanding of a particular issue. Primarily, the sample of the study comprises staff and officials of PHC. The following criteria were used to select the respondents:

Personnel who have been associated with the creation of $\mathrm{PHC}$ and its anti-quackery mandate in particular. Personnel who are or have been associated with the implementation of the anti-quackery mandate of PHC

A semi-structured interview guide, based on a review of existing literature, was adopted to ensure responses remained relevant and new strategies of inquiry could be explored as needed. Key Informant Interviews (KIIs) were conducted, beginning with questions regarding sociodemographic characteristics, subsequently moving towards more specific domains of inquiry related to health service regulation, $\mathrm{PHC}$ and its anti-quackery mandate. Duration of each interview was kept at moderated length and key ethical considerations were observed.

Data was analyzed by using an inductive as well deductive approach and revealed both pre-determined and emergent themes. In the first step of analysis, audio-visual recordings were transcribed and reviewed extensively for identification of important themes and ideas. Codes were developed on the basis of similarities of responses, which were then divided thematically into broader categories to formulate findings.

\section{RESULTS}

This section of the study provides an overview of key findings drawn from the analysis of respondents' data. The major themes that emerged are as follows:

Lack of Political and Administrative Will: One of the most important barriers that hinders the progress of the Anti-Quackery Cell within the broader structure of $\mathrm{PHC}$ is a lack of political and administrative will.

The political setup of Pakistan is often unstable and short term, which makes the execution of policy difficult ${ }^{10}$. Findings suggested that political leadership wasless concerned with eliminating the menace of quackery and was disproportionately focused on other agendas, such as universal health provision and reproductive health. One of the respondents shared:
"In Pakistan, politicians are leastinterested in eradicating quackery and most of the policies are framed with afocus on other domains of health care system. Lack of political will is one of the core hurdlesto get rid of quackery in the Country."

Similarly, administrative will i.e. lack of interest by the higher ups of PHC and Anti-Quackery Cell, was a common concern among respondents. It was found that frequent transfer postings of theAdministrative Head of $\mathrm{PHC}$ and Anti-Quackery Cell have a severe impact on the implementation of effective and timely policies. This casts a two-pronged negative effect; in the first place the Administrative Head lacks a pertinent interest in the antiquackery framework, as a result of which lower staff's motivation and dedication goes down. One of the interviewees stated:

"I have observed during my tenureatthe Anti-Quackery Cell that no Head of the Department remained on the seat for a long period. Such situations make it difficult for us to focus on the tasks assigned. Each officer comes in with a particular mind-set, which is why a change in leadership changes the entire roadmap and process."

Therefore, the study found that lack of political and administrative will isa serious problem and barrier towards the eradication of quackery in Pakistan.

Critical Decision-Making at the Top Level: Another important challenge that negatively impacts PHC's antiquackery framework was the lack of and/or delayed decision-making at the top level. For the progress and development of any policy, particularly a policy related to health, timely decision-making is critical ${ }^{11}$. For a comprehensive approach, both strategic and operational decision-making must be seamlessly integrated and directed towardsthe mandate of healthcare policies.

Findings show that with reference to its anti-quackery policy, PHC lacks strategic, operational and timely decision-making by higher authorities. This directly influences the prevalence of quackery in Pakistan. One of the respondents highlighted:

"The delay in taking action results in discouragement of people who report establishments run by quacks. As it is, people in general don't have faith in the enforcement of regulations in any sector in our Country."

Hence, the study underlinesthat lack of timely and critical decision-making at the top level is a major problem for the mandate of the Anti-quackery Cell.

Untrained staff and inappropriate HR selection: The success of a healthcare system depends significantlyupon the professional competency of its staff. Analysis of existing literature suggested that professional training increases productivity and progress towards achieving the objectives of healthcare policy ${ }^{12}$.

In this vein, findings illustratethat the Anti-Quackery Cell lacks professional and trained staff. Such incompetent human resource does not work well in the field, thereby limiting progress made on curbing of quackery. A PHC field official shared:

"All my knowledge about dealing with quacks in my area was gained on the job. When I was a new recruit several years ago, no one taught me how to identify and take action against these menaces." 
This observation shows that most of the officials involved in anti-quackery did not have sufficient knowledge about field operations. Furthermore, there is a dire need of continued professional development to ensure staff is able to perform their duties efficiently.

In addition to the untrained staff, the process of recruitment of related staff is also an important factor that impacts the performance of the Anti-Quackery Cell. Findings suggest thatPHC's staffing processlacks relevance. Most of the officials recruited to perform their duties in the Cell did not have relevant degrees. An official of the PHC cell pointed out:

"Take for example, the Assistant Manager Anti-Quackery and Document Controller, who have graduate degrees in Gender Studies and Commerce respectively."

Similarly, most Deputy District Health Officers (DDHOs)were medical graduates with no prior experience dealing with quacks. This continually reflects in errors made in the handling and reporting of medicines or other perishable items found during sealing of premises. Hence, relevance of staff, as well as lack of trained staff, is a major challenge in the successful implementation of antiquackery in Pakistan.

Lack of legislation regarding anti-quackery: Initially, the Anti-Quackery Cell was established via the "Standing Orders of the Punjab Healthcare Commission for banning quackery in all its forms and manifestations and for dealing with quacks". This establishment was later canceled by the Lahore High Court, with the PHC ordered to frame proper legislation for the said cell. As a result, some laws were framed, but they lacked strategic as well as operational relevance. Furthermore, it has been observed that most of the operational domains of the Anti-Quackery Cell lack legislation. One field officer shared:

"We lack procedural guidelines when to seal, de-seal and capture assets. Similarly, the types of assets that have to be captures need to be defined. Such issues make our procedure unnecessarily difficult and less fruitful."

Hence, the study found that lack of proper legislation is an important challenge for the framework of anti-quackery and allows quacks to use loopholes in existing laws to continue their practices.

Insufficient organizational arrangements: Findings highlight that the Anti-Quackery Cell lacked organizational arrangements. DDHOs, who were directed to execute the tasks of anti-quackery, were not provided adequate staff or offices. One of the DDHOs said:

"I feel like a donkey who is expected to simply slave away all day and never complain. I have no 'working hours' - the duty never ends. I have no space to sit, conduct meetings or take stock of the day's activities. I don't think the Government has any regard for my time or effort because I am treated like I have no dignity."

Similarly, field staff lacked security staff and did not have sufficient recourses to store material confiscated from quacks. This may further pose a serious problem when coordinating with the District administration, as well as toplevel officials of PHC.

Therefore, this study emphasizes weak organizational arrangements for anti-quackery staff as one of the factors hampering the successful elimination of quackery.
Deep Roots of Quackery in Pakistan: Findings suggest that quackery is deeply rooted within the cultural context of Pakistan. The practice is not expensive and has wideranging scope and accessibility. This explains why there are quacks in every nook and corner of the country, whereas specialized doctors are rarely available in faraway areas. One of the respondent highlighted:

"Quackery has been most widely practiced field in our Country for a long time. During field visits, we often encounter resistance, as the local community prefers quacks and provides shelter to them"

Hence, it is difficult to deal with an issue that is so deeply ingrained in society and favors the cultural context as well.

\section{DISCUSSION}

Findings of the study show that there are multiple challenges to curb the issue of quackery in Pakistan. Lack of political and administrative will, inappropriate staff, lack of training of employees are key issues that make it difficult to eliminate this practice from society ${ }^{13}$.

Furthermore, it is observed that most of the findings of the study were aligned with the results that emerged in existing literature. Regulations regarding anti-quackery are often less beneficial as there is lack of political and administrative will ${ }^{14}$. Similarly, professionals responsible for eliminating quackery are often incompetent and have fewer in-service trainings, which further limits the progress of the anti-quackery agenda in Pakistan and other developing Countries.

Many studies support the finding that quackery is deeply rooted in the cultural practices of our region.In Pakistan alternative therapies have been availed by people who have faith in clergymen, spiritual healers, homeopaths, homeopaths and even quacks. These are the preferred choice for ailments such as epilepsy, psychosomatic troubles, epilepsy, depression and several other problems. Indigenous medicine is a significant source of health care, specifically in tribal and rural areas of the country. The prime reasons for seeking consultation from an alternative medicine practitioner is the modest fee, proximity, family pressure, availability and the community's strong approval. There is very rich tradition of using medicinal plants for the treatment of all kinds of illnesses ${ }^{15}$. In another study it is stated that quackery in Pakistan is considered a norm. Spiritual healing, mental healing and unlicensed practice of prescribing medicine are common sights ${ }^{16}$.

\section{CONCLUSION}

The study concludes that quackery has deeproots in Pakistan as it is well-suited to the socio-economic reality of the Country. It serves as a less-expensive alternate to the professional healthcare system.

Serious efforts have been made to control this menace, as it is playing havoc with the lives of individuals and system of society. PHC formulated a plan and organizational structure in the name of the Anti-Quackery Cell to control quackery, but the results are not satisfactory.

There are numerous challenges that hamper the success of the Anti-Quackery Cell. These challenges are twofold i.e. issues at the higher level and lower level. At the higherlevel, lack of political and administrative will, along with the recruitment irrelevance and lack of legislation, are 
important factors. At the lower level, lack of professional development and the deep-rooted nature of quackery in society are major challenges to control quackery in Pakistan.

Disclaimer: This research received no grant from any funding agency in the public, commercial, or not-for-profit sectors and there is no conflict of interest.

\section{REFERENCES}

1. Datta R. The world of quacks: a parallel health care system in rural West Bengal. IOSR Journal of Humanities and Social Science 2003; 14(2): 44-53.

2. Ghaffar A, Kazi BM, Salman M. Health care systems in transition III. Pakistan, Part I. An overview of the health care system in Pakistan. Journal of Public Health 2000; 22(1): 3842.

3. George A, lyer A.Unfree markets: socially embedded informal health providers in northern Karnataka, India. Social Science \& Medicine 2013; 96: 297-304.

4. Punjab Healthcare Commission. PHC Anti Quackery Strategy 2013; 32(1)

5. Norcross JC, KoocherGP, Garofalo A. Discredited psychological treatments and tests: a Delphi poll. Professional Psychology: Research and Practice 2006; 37(5): 515.

6. Shahzad $H$, Farnaz $M$, Humayun $R$, Muhammad $A Q$, Nadeem K. Alternative and Traditional Medicines Systems in Pakistan: History, Regulation, Trends, Usefulness, Challenges, Prospects and Limitations. INTECH Open Access Publisher; 2012.
7. Mok EA, Gostin LO, Gupta MD, Levin M. Implementing public health regulations in developing countries: lessons from the OECD countries. The Journal of Law, Medicine \& Ethics 2010; 38(3): 508-519.

8. Bruch H, Ghoshal S. A bias for action: How effective managers harness their willpower, achieve results, and stop wasting time. Harvard Business Press 2004.

9. Newman LW. Social research methods (4th Ed.). Boston: Allyn and Bacon, 2000.

10. Talati JJ, Pappas G. Migration, medical education, and health care: a view from Pakistan. Acad Med 2006; 81 (12): 55-62.

11. Tatum BC, Eberlin R, Kotttraba C, Bradberry T. Leadership, decision making, and organizational justice. Management Decision 2003; 41(10): 1006-1016.

12. Dysinger WS, King V, Foster TC, Geffken D.Incorporating population medicine into primary care residency training. Fam Med 2011;43(7):480-486.

13. Lang TA, Hodge $M$, Olson V. Nurse-patient ratios: A systematic review on the effects of nurse staffing on patient, nurse employee, and hospital outcomes. J Nurs Adm 2004; 34: 326-37.

14. RAND Corporation. International comparison of ten medical regulatory systems. Rand Corporation 2009.

15. Shaikh BT, Hatcher J. Complementary and alternative medicine in Pakistan: prospects and limitations. EvidenceBased Complementary and Alternative Medicine2005; 2(2): 139-142.

16. Hussain S, Malik F, Hameed A, Ahmed S, Riaz H, Abbasi N, et al. Pakistani Pharmacy Students' Perception About Complementary and Alternative Medicine. American Journal of Pharmaceutical Education 2012;76(2):21. 